\title{
On the difference between bridge rules and lifting axioms
}

\author{
P. Bouquet ${ }^{1,2}$ and L. Serafini ${ }^{2}$ \\ ${ }^{1}$ Department of Information and Communication Technologies - University of Trento \\ Via Inama, 5 - 38100 Trento (Italy) \\ ${ }^{2}$ ITC-IRST - Istituto per la Ricerca Scientifica e Tecnologica \\ Via Sommarive - 38050 Trento (Italy) \\ bouquetecs.unitn.it serafini@itc.it
}

\begin{abstract}
In a previous paper, we proposed a first formal and conceptual comparison between the two most important formalizations of context in AI: Propositional Logic of Context (PLC) and Local Models Semantics/MultiContext Systems (LMS/MCS). The result was that LMS/MCS is at least as general as PLC, as it can be embedded into a particular class of MCS, called MPLC. In this paper we go beyond that result, and prove that, under some important restrictions (including the hypothesis that each context has finite and homogeneous propositional languages), MCS can be embedded in PLC with generic axioms. To prove this theorem, we prove that MCS cannot be embedded in PLC using only lifting axioms to encode bridge rules. This is an important result for a general theory of context and contextual reasoning, as it proves that lifting axioms and entering context are not enough to capture all forms of contextual reasoning that can be captured via bridge rules in LMS/MCS.
\end{abstract}

\section{Introduction}

This paper continues the investigation of formal theories of context we started in [3]. In that paper, we compared two well-known formalizations of context, namely the Propositional Logic of Context (PLC) [5] and Local Models Semantics (LMS) [7], axiomatized via Multi Context Systems $[9,8](\mathrm{MCS})^{1}$. The main technical result was that LMS/MCS is at least as general as PLC, as it can be embedded into a particular class of MCS, called MPLC.

In this paper we go beyond that result, and analyze the claim that LMS/MCS is strictly more general than PLC. The main technical results are the following: (i) under some important restrictions (including the hypothesis that each context has finite and homogeneous propositional languages), LMS/MCS can be embedded in PLC with generic axioms; (ii) LMS/MCS cannot be embedded in PLC using only lifting axioms to encode bridge rules. These results are important for a general theory of context and contextual reasoning in two senses: first, the restrictions needed to prove the first theorem have a significant impact on the fulfillment of the intuitive desiderata that were

\footnotetext{
${ }^{1}$ Hereafter, we will refer to the general framework of LMS together with its axiomatization via MCS as LMS/MCS.
} 
brought forward to motivate the formalization of context in AI (e.g., in [11]); second, they prove that lifting axioms and entering context are not enough to capture all forms of contextual reasoning that can be captured via bridge rules in LMS/MCS.

\section{The two systems: PLC and LMS/MCS}

In this section we quickly revise the two formalisms, and prepare the ground for the technical comparison between them ${ }^{2}$.

\subsection{Propositional Logic of Context}

In this paper, we use the version of PLC presented in [4]. Given a set $\mathbb{K}$ of labels, intuitively denoting contexts, the language of PLC is a multi modal language on a set of atomic propositions $\mathbb{P}$ with the modality $i s t(\kappa, \phi)$ for each context (label) $\kappa \in \mathbb{K}$. More formally, the set of well formed formulae $\mathbb{W}$ of PLC, based on $\mathbb{P}$, are

$$
\mathbb{W}:=\mathbb{P} \cup(\neg \mathbb{P}) \cup(\mathbb{P} \supset \mathbb{P}) \cup \operatorname{ist}(\mathbb{K}, \mathbb{P})
$$

The other propositional connectives are defined as usual. If $\kappa$ is a context, then the formula $i s t(\kappa, \phi)$ can be read as: $\phi$ is true in the context $\kappa$. PLC allows to describe how a context is viewed from another context. For this PLC introduces sequences of contexts (labels). Let $\mathbb{K}^{*}$ denote the set of finite contexts sequences and let $\bar{\kappa}=\kappa_{1} \ldots \kappa_{n}$ denote any (possible empty) element of $\mathbb{K}^{*}$. The sequence of contexts $\kappa_{1} \kappa_{2}$ represents how context $\kappa_{2}$ is viewed from context $\kappa_{1}$. Therefore, the intuitive meaning of the formula $i s t\left(\kappa_{2}, \phi\right)$ in the context $\kappa_{1}$ is that $\phi$ holds in the context $\kappa_{2}$, from the point of view of $\kappa_{1}$. Similar interpretation can be given to formulae in sequences of contexts longer than 2. A model for PLC associates a set of partial truth assignments to a subset of context sequences and satisfiability is defined with respect to a context sequence.

Definition 1. A model $\mathfrak{M}$ of PLC is a partial function which maps context sequences in $\mathbb{K}^{*}$ into a set of partial truth assignments for $\mathbb{P}$.

$$
\mathfrak{M} \in\left(\mathbb{K}^{*} \rightarrow p \mathbf{P}(\mathbb{P} \rightarrow p\{\text { true }, \text { false }\})\right)
$$

where $A \rightarrow p$ denotes the set of partial functions from $A$ to $B$ and $\mathbf{P}(A)$ denotes the powerset of $A$.

The original intuition was that, partial truth assignments allow us to represent the fact that in different context sequences there are different sets of meaningful formulae. Indeed, a model $\mathfrak{M}$ defines a vocabulary, denoted by $\operatorname{Vocab}(\mathfrak{M})$, namely, a function that associates to each context sequence a set of meaningful formulae. Formally, a vocabulary is a relation $\mathrm{Vocab} \subseteq \mathbb{K}^{*} \times \mathbb{P}$ that associates a subset of primitive propositions with each context. $\operatorname{Vocab}(\mathfrak{M})$, i.e, the vocabulary defined by the model $\mathfrak{M}$, is the function that associates to each context sequence $\bar{\kappa}$ a subset of $\mathbb{P}$ for which all the assignments in

\footnotetext{
${ }^{2}$ An exhaustive presentation of the two formalisms is beyond the scope of this paper; interested readers can refer to the bibliography for more details.
} 
$\mathfrak{M}(\bar{\kappa})$ are defined. That is, $\langle\bar{\kappa}, p\rangle \in \operatorname{Vocab}(\mathfrak{M})$ if and only if $\mathfrak{M}(\bar{\kappa})$ is defined and, for all $\boldsymbol{v} \in \mathfrak{M}(\bar{\kappa}), v(p)$ is defined (where $v$ is a truth assignment to atomic propositions).

Satisfiability and validity of formulae are defined only for these models that provides enough vocabulary, i.e. the vocabulary which is necessary to evaluate a formula in a context sequence. Each formula $\phi$ in a context sequence $\bar{\kappa}$ implicitly defines its vocabulary, denoted by $\operatorname{Vocab}(\bar{\kappa}, \phi)$, which intuitively consists of the minimal vocabulary necessary to build the formula $\phi$ in the context sequence $\bar{\kappa}$. More formally, $\operatorname{Vocab}(\bar{\kappa}, \phi)$ is recursively defined as follows:

$$
\begin{aligned}
\operatorname{Vocab}(\bar{\kappa}, p) & =\{\langle\bar{\kappa}, p\rangle\} \\
\operatorname{Vocab}(\bar{\kappa}, \neg \phi) & =\operatorname{Vocab}(\bar{\kappa}, \phi) \\
\operatorname{Vocab}(\bar{\kappa}, \phi \supset \psi) & =\operatorname{Vocab}(\bar{\kappa}, \phi) \cup \operatorname{Vocab}(\bar{\kappa}, \psi) \\
\operatorname{Vocab}(\bar{\kappa}, i s t(\kappa, \phi)) & =\operatorname{Vocab}(\bar{\kappa} \kappa, \phi)
\end{aligned}
$$

Definition 2 (Satisfiability and Validity). Let $\phi$ and $\mathfrak{M}$ be a formula and a model respectively. $\phi$ is satisfied in $\mathfrak{M}$ by an assignment $\boldsymbol{v} \in \mathfrak{M}(\bar{\kappa})$ (notationally $\mathfrak{M}, \boldsymbol{\nu}=_{\bar{\kappa}} \phi$ ) according to the following clauses:

1. $\mathfrak{M}, \boldsymbol{v}=\overline{\bar{\kappa}} p$ iff $\boldsymbol{v}(p)=$ true;

2. $\mathfrak{M}, \boldsymbol{v}==_{\overline{\mathrm{K}}} \neg \phi$ iff not $\mathfrak{M}, \boldsymbol{v} \models_{\overline{\mathrm{K}}} \phi$;

3. $\mathfrak{M}, \boldsymbol{v}={ }_{\overline{\mathrm{K}}} \phi \supset \psi$ iff not $\mathfrak{M}, \boldsymbol{v}=_{\overline{\mathrm{K}}} \phi$ or $\mathfrak{M}, \boldsymbol{v}={ }_{\overline{\mathrm{K}}} \psi$;

4. $\mathfrak{M}, \boldsymbol{v}={ }_{\bar{\kappa}}$ ist $(\kappa, \phi)$ iff for all $\boldsymbol{v}^{\prime} \in \mathfrak{M}(\bar{\kappa} \kappa), \mathfrak{M}, v^{\prime}={ }_{\bar{\kappa} \kappa} \phi$;

5. $\mathfrak{M}=_{\overline{\mathrm{K}}} \phi$ iff for all $\boldsymbol{v} \in \mathfrak{M}(\overline{\mathrm{\kappa}}) ; \mathfrak{M}, \boldsymbol{v} \models_{\overline{\mathrm{K}}} \phi$;

6. $\models_{\bar{\kappa}} \phi$ iff for all PLC-model $\mathfrak{M}$, such that $\operatorname{Vocab}(\bar{\kappa}, \phi) \subseteq \operatorname{Vocab}(\mathfrak{M}), \mathfrak{M}=\bar{\kappa} \phi$.

$\phi$ is valid in a context sequence $\overline{\mathrm{K}}$ if $\left.\right|_{\overline{\mathrm{\kappa}}} \phi$; $\phi$ is satisfiable in a context sequence $\overline{\mathrm{\kappa}}$ if there is a PLC-model $\mathfrak{M}$ such that $\mathfrak{M}=\bar{\kappa} \phi$. A set of formulae $T$ is satisfiable at a context sequence $\bar{\kappa}$ if there is a model $\mathfrak{M}$ such that $\mathfrak{M} \models_{\bar{\kappa}} \phi$ for all $\phi \in T$.

According to the above definition, vocabularies affect truth in contexts making each formula outside the vocabulary false. This implies that a PLC-model $\mathfrak{M}$ presents a non classical semantics for all the formulas $\phi$ such that $\langle\bar{\kappa}, \phi\rangle \nsubseteq \operatorname{Vocab}(\mathfrak{M})$. For instance, if a proposition $\langle\bar{\kappa}, p\rangle \notin \operatorname{Vocab}(\mathfrak{M})$ then $\mathfrak{M} \not \nvdash_{\bar{\kappa}} p \vee \neg p$. This "non classical" effect however disappear in the definition of validity. For validity of a formula $\phi$ is checked by considering only the models whose vocabularies contain $\phi$. This means that validity and satisfiability can be formulated by considering only PLC-models with complete vocabularies, i.e. PLC-models $\mathfrak{M}$ 's with $\langle\bar{\kappa}, p\rangle \in \operatorname{Vocab}(\mathfrak{M})$ for each $p \in \mathbb{P}$ and $\bar{\kappa} \in \mathbb{K}^{*}$.

Theorem 1 (Reduction to complete vocabulary). A formula is valid in PLC if and only if it is satisfied by all the PLC-models with complete vocabulary. Similarly, a formula is satisfiable in PLC if and only if there is a PLC-model with complete vocabulary that satisfies it.

Ignoring vocabularies, PLC is a multi-modal K extended with the axiom $(\Delta)$, on the set of propositions $\mathbb{P}$. Indeed the Hilbert style axiomatization of validity proposed in [4] presented in Figure 1 -is the modal system K extended with the axiom $(\Delta)$. 
(PL) $\vdash_{\bar{\kappa}} \phi \quad$ If $\phi$ is an instance of a classical tautology

$(\mathrm{K}) \vdash_{\bar{\kappa}} i s t(\kappa, \phi \supset \psi) \supset \operatorname{ist}(\kappa, \phi) \supset \operatorname{ist}(\kappa, \psi)$

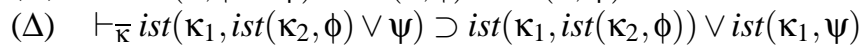

(MP) $\frac{\vdash_{\bar{\kappa}} \phi \quad \vdash_{\bar{\kappa}} \phi \supset \psi}{\vdash_{\bar{\kappa}} \psi}$
(CS) $\frac{\vdash_{\bar{\kappa} \kappa} \phi}{\vdash_{\bar{\kappa}} i s t(\kappa, \phi)}$

Fig. 1. Axioms and inference rules for PLC

\subsection{Local Models Semantics and Multi-Context Systems}

The version of LMS we present here was presented in [7]. Let $\left\{L_{i}\right\}_{i \in I}$ be a family of languages defined over a set of indexes $I$ (in the following we drop the index $i \in I$ ). Intuitively, each $L_{i}$ is the (formal) language used to describe the facts in the context $i$. In this paper, we assume that $I$ is (at most) countable. Let $M_{i}$ be the class of all the models (interpretations) of $L_{i}$. We call $m \in M_{i}$ a local model (of $L_{i}$ ).

To distinguish the formula $\phi$ occurring in the context $i$ from the occurrences of the "same" formula $\phi$ in the other contexts, we write $i: \phi$. We say that $i: \phi$ is a labelled wff, and that $\phi$ is an $L_{i}$-wff. For any set of labeled formulae $\Gamma, \Gamma_{i}=\{\phi \mid i: \phi \in \Gamma\}$.

Definition 3 (Compatibility chain $^{3}$ ). A compatibility chain $\mathbf{c}=\left\{c_{i} \subseteq M_{i}\right\}_{i \in I}$ is a family of set of models of $L_{i}$ such that each $c_{i}$ is either empty or a singleton. We call $c_{i}$ the $i$-th element of $\mathbf{c}$. A compatibility chain is nonempty if one of its components is nonempty.

A compatibility chain represents a set of "instantaneous snapshots of the world" each of which is taken from the point of view of the associated context. Due to the fact that contexts describe points of view of the same world, certain combinations of snapshots are possible while others can never happen. To distinguish between these two sets, LMS contains the notion of compatibility relation-defined in the followingrepresents the "admissible" combinations snapshots.

Definition 4 (Compatibility relation and LMS-model). A compatibility relation is a set of compatibility chains. A LMS-model is a compatibility relation that contains a nonempty compatibility chain.

Definition 5 (Satisfiability and Entailment). Let $=$ be the propositional classical satisfiability relation. We extend the definition of $\mid=$ as follows:

1. for any $\phi \in L_{i}, c_{i}=\phi$ if, for all $m \in c_{i}, m=\phi$;

2. $\mathbf{c}=i: \phi$ if $c_{i} \models \phi$;

3. $\mathbf{C}=i: \phi$ if, for all $\mathbf{c} \in \mathbf{C}, \mathbf{c}=i: \phi$;

4. $\Gamma_{i} \models{ }_{c_{i}} \phi$ if, for all $m \in c_{i}$, if $m \models \Gamma_{i}$, then $m \models \phi$;

\footnotetext{
${ }^{3}$ For the sake of this paper, we use a definiton of compatibility chain which is specialized and simpler than the one given in [7].
} 
5. $\Gamma \models \mathbf{c} i: \phi$ if, either there is a $j \neq i$, such that $c_{j} \not \models \Gamma_{j}$, or $\Gamma_{i} \models_{c_{i}} \phi$;

6. $\Gamma \models_{\mathbf{C}} i: \phi$ if, for all $\mathbf{c} \in \mathbf{C}, \Gamma \models \mathbf{c} i: \phi$;

7. For any class of models $\mathfrak{C}, \Gamma \models_{\mathfrak{C}} i: \phi$ if, for all models $\mathbf{C} \in \mathfrak{C}, \Gamma \models_{\mathbf{C}} i: \phi$.

We adopt the usual terminology of satisfiability and entailment for the statements about the relation $\models$. Thus we say that $\mathbf{c}$ satisfies $\phi$ at $i$, or equivalently, that $\phi$ is true in $c_{i}$, to refer to the fact that $c_{i}=\phi$. We say that $\Gamma$ entails $i: \phi$ in $\mathbf{c}$ to refer to the fact that $\Gamma \models_{\mathbf{c}} i: \phi$. Similar terminology is adopted for $\Gamma \models_{\mathbf{C}} i: \phi$ and $\Gamma \models_{\mathfrak{C}} i: \phi$.

MultiContext Systems (MCS) [9] are a class of proof systems for $\mathrm{LMS}^{4}$. The key notion of an MCS is that of bridge rule.

Definition 6 (Bridge Rule). A bridge rule on a set of indices I is a rule of the form:

$$
\frac{i_{1}: \phi_{1} \quad \ldots \quad i_{n}: \phi_{n}}{i: \phi} b r
$$

where $i_{1}, \ldots, i_{n}, i \in I$, A bridge rule can be associated with a restriction, namely a criterion which states the conditions of its applicability.

Definition 7 (MultiContext System (MCS)). A MultiContext System for a family of languages $\left\{L_{i}\right\}$, is a pair $\mathrm{MS}=\left\langle\left\{C_{i}=\left\langle L_{i}, \Omega_{i}, \Delta_{i}\right\rangle\right\}, \Delta_{b r}\right\rangle$, where each $C_{i}=\left\langle L_{i}, \Omega_{i}, \Delta_{i}\right\rangle$ is a theory (on the language $L_{i}$, with axioms $\Omega_{i}$ and natural deduction inference rules $\left.\Delta_{i}\right)$, and $\Delta_{b r}$ is a set of bridge rules on $I$.

MCSs are a generalization of Natural Deduction (ND) systems [12]. The generalization amounts to using formulae tagged with the language they belong to. This allows for the effective use of the multiple languages. The deduction machinery of an MCS is the composition of two kinds of inference rules: local rules, namely the inference rules in each $\Delta_{i}$, and bridge rules. Local rules formalize reasoning within a context (i.e. are only applied to formulae with the same index), while bridge rules formalize reasoning across different contexts.

Deductions in a MCS are trees of formulae which are built starting from a finite set of assumptions and axioms, possibly belonging to distinct languages, and by a finite number of application of local rules and bridge rules.

\subsection{Lifting axioms and bridge rules}

A crucial feature of a formal theory of context—contained both in LMS/MCS and PLC - is the possibility to specify relations between facts of different contexts. This is an essential feature of contextual reasoning, as contexts are not simply unrelated representations, but typically are different representations of the same world. For example, two contexts may describe the same piece of the world from the same perspective, but at different level of detail; or may describe the same piece of the world, only from different perspectives. PLC formalizes relations between contexts via lifting axioms, while LMS/MCS uses bridge rules. Lifting axioms are defined as

\footnotetext{
${ }^{4}$ In this paper, we present a definition of MC system which is suitable for our purposes. For a fully general presentation, see [9].
} 
"... axioms which relate the truth in one context to the truth in another context. Lifting is the process of inferring what is true in one context based on what is true in another context by the means of lifting axioms" [10]

The general form of lifting axioms is the following:

$$
i s t\left(\kappa_{1}, \phi_{1}\right) \wedge \ldots \wedge i s t\left(\kappa_{n}, \phi_{n}\right) \supset i s t(\kappa, \phi)
$$

As any formula in PLC, lifting axioms must be stated in a context. The lifting axiom above can be intuitively read as " $\phi$ is true in a context $\kappa$ if the formulas $\phi_{1}, \ldots, \phi_{n}$ are true in the contexts $\kappa_{1}, \ldots, \kappa_{n}$ respectively".

Bridge rules, introduced in Definition 6, are inference rules whose premises and conclusion belong to different contexts. The general form of bridge rules is described in [9], and can be though as a generalization of a Natural Deduction inference rules [12] which involve more than one index. For the sake of this paper we consider only bridge rules of the following form.

$$
\frac{\kappa_{1}: \phi_{1} \quad \ldots \quad \kappa_{n}: \phi_{n}}{\kappa: \phi} b r
$$

The above bridge rules roughly formalize the same intuition as that formalized by lifting axiom (1).

The main difference between lifting axioms and bridge rules is that lifting axioms are stated in an external context, which must be expressive enough to represent facts of all the contexts involved (using ist-formulae), whereas bridge rules allow stating relations between contexts without the need of an external context. There are situations where having an external context may be an advantage (for example, when one needs to reason about lifting axioms themselves, e.g. to discover that a lifting axiom is redundant, or leads to inconsistent contexts). However, in general, specifying an external context can be very costly - especially when there are many interconnected contexts - as the external context essentially duplicates the information of each context. LMS/MCS allows both solutions. Indeed, instead of using bridge rules to lift a fact $\phi$ from $\kappa_{1}$ to $\kappa_{2}$, one can define a third context connected with $\kappa_{1}$ and $\kappa_{2}$ via bridge rules and explicitly add an axiom like (1) to this new context ${ }^{5}$. This very last observation constitutes the underlying idea of the proof of the fact that PLC can be embedded in LMS/MCS described in [3]. The converse question, i.e., if LMS/MCS can be reconstructed in PLC will be answered in the rest of this paper. As a consequence we will have a sharper intuition on the analogies and differences between bridge rules and lifting axioms.

\section{Reconstructing LMS/MCS in PLC}

Since a comparison of the two logical systems should be done on a common ground, we consider LMS/MCS with homogeneous languages, i.e., LMS/MCS whose contexts have all the same propositional language. Indeed, as it is shown by Treorem 1, PLC does

5 This approach was used, for example, in the solution to the qualification problem presented in [2]. 

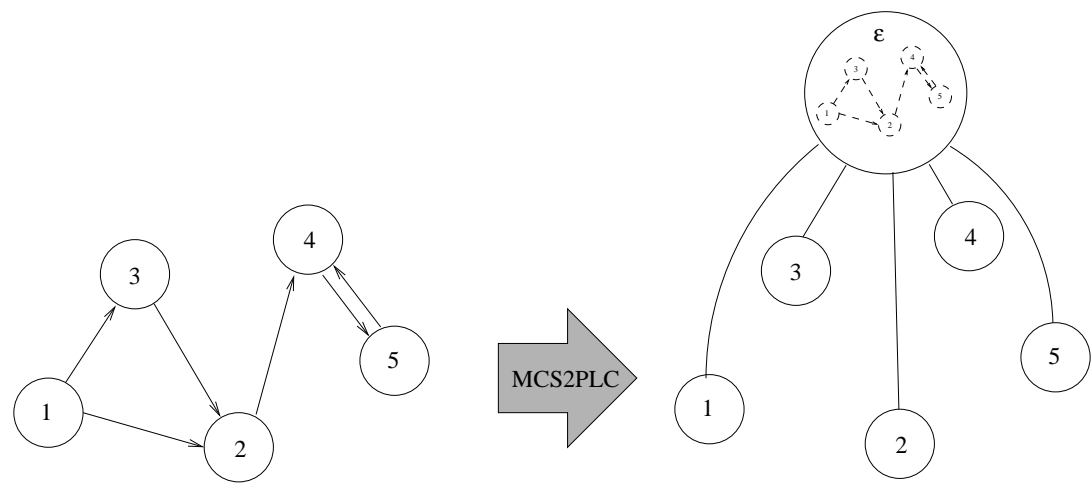

Fig. 2. Embedding LMS/MCS into PLC

not support contexts with different languages. Similarly we restrict the comparison to LMS/MCS in which all contexts have the same inference engine, which is contexts are all classical propositional theories.

The general intuition for encoding an MCS into PLC is shown in Figure 2. Given a MCS with $I$ contexts, we define a PLC with $I$ contexts (one for each context in MCS) and an additional (meta/external)-context $\varepsilon$. The content of each context in $I$ and the compatibility relations (bridge rules) between contexts are described via ist-formulas in in $\varepsilon$. The representation of the content of the MCS contexts is quite straightforward: any formula $i: \phi$ in MCS is translated into a formula $\varepsilon$ : ist $(i, \phi)$ in PLC. For bridge rules, the translation is more tricky. Indeed, the intuition that a bridge rule like (2) is translated into the lifting axiom (1) does not work. Indeed, the following theorem proves a first important fact, namely that in general bridge rules cannot be modeled in PLC only as a set of lifting axioms. Let $\mathbb{B R}_{I}$ be the set of bridge rules between a set $I$ of contexts with language $L_{i}=L_{j}$ (for $i, j \in I$ ).

Let $\mathbb{L} \mathbb{A} \subset \mathbb{W}$ the set of lifting axioms among the contexts $I$ expressed in a new context $\varepsilon$ not in $I$. The notation $\Gamma \vdash_{b r} i: \phi$ stands for: $i: \phi$ is derivable from $\Gamma$ in the MCS with the set $I$ of contexts, no axioms, and the set $\boldsymbol{b} \boldsymbol{r}$ of bridge rules.

Theorem 2. There is no transformation la $: \mathbb{B} \mathbb{R} \rightarrow \mathbb{L} \mathbb{A}$ such that for any finite subset br $\subseteq \mathbb{B R}$ of bridge rules:

$$
\begin{gathered}
i_{1}: \phi_{1}, \ldots, i_{n}: \phi_{n} \vdash_{\text {br }} i: \phi \\
\text { if and only if } \\
\vdash_{\varepsilon} \wedge_{\text {br } \in \text { br }} l a(b r) \supset\left(\text { ist }\left(i_{1}, \phi_{1}\right) \wedge \ldots \wedge \text { ist }\left(i_{n}, \phi_{n}\right) \supset \operatorname{ist}(i, \phi)\right)
\end{gathered}
$$

Proof. The theorem is proved by counterexample. Consider the following two bridge rules.

$$
\frac{1: p}{2: q} b r_{12} \quad \frac{2: q}{1: r} b r_{21}
$$

where $p, q$, and $r$ are three distinct propositional letters. Let $b r_{12}$ and $b r_{21}$ be both unrestricted (i.e., always applicable). Considering $b r_{12}$ or $b r_{21}$ separately, they do not 
affect theoremhood in either context 1 and 2. Formally, for $i=1,2, \vdash_{b r_{12}} i: \phi$ if and only if $\phi$ is a propositional tautology, and analogously $\vdash_{b r_{21}} i: \phi$ if and only if $\phi$ is a tautology (see [6] for a proof of a similar fact). Instead, combining $b r_{12}$ and $b r_{21}$ in the same MCS, new theorems, which are not tautologies, can be proved. An example of such a theorem is $1: p \supset r$, and its proof is the following:

$$
\frac{\frac{1: p^{(*)}}{2: q} b r_{12}}{\frac{1: r}{1: p \supset r} r_{21} \supset \mathrm{I}\left(\text { Discharging the assumption }^{(*)}\right)}
$$

Let $l a\left(b r_{12}\right)$ and $l a\left(b r_{21}\right)$ be the following general conjunctions of lifting axioms:

$$
\begin{aligned}
& l a\left(b r_{12}\right)=\bigwedge_{m=1}^{M}\left(\bigwedge_{k=1}^{K_{m}} i \operatorname{sit}\left(i_{m k}, \phi_{m k}\right) \supset \operatorname{ist}\left(j_{m}, \psi_{m}\right)\right) \\
& l a\left(b r_{21}\right)=\bigwedge_{n=M+1}^{N}\left(\bigwedge_{k=1}^{K_{n}} i s t\left(i_{n k}, \phi_{n k}\right) \supset \operatorname{ist}\left(j_{n}, \psi_{n}\right)\right)
\end{aligned}
$$

where $i_{m k}, i_{n k}$, and $j_{n}$ are either 1 or 2 . Posing $\boldsymbol{b r}=\left\{b r_{12}, b r_{21}\right\}$, we have that $\bigwedge_{b r \in \boldsymbol{b r}} l a(b r)$ is equivalent to the following formula:

$$
\bigwedge_{n=1}^{N}\left(\bigwedge_{k=1}^{K_{n}} i s t\left(i_{n k}, \phi_{n k}\right) \supset i s t\left(j_{n}, \psi_{n}\right)\right)
$$

Suppose, for contradiction, that equivalence (3) holds. Since $1: p \supset r$ is derivable via $b r_{12}$ and $b r_{21}$, we have that

$$
\vdash_{\varepsilon} \bigwedge_{b r \in \boldsymbol{b r}} l a(b r) \supset i s t(i, p \supset r)
$$

Consider the PLC-model $\mathfrak{M}$ with $\mathfrak{M}(1)$ equal to all the assignments for $L_{1}$ and $\mathfrak{M}(2)$ equal to all the assignments for $L_{2}$. Since $p \supset r$ is not valid, there is an assignment $v$ such $\vee \not \models p \supset r$. By construction, $\mathfrak{M}(1)$ contains all the assignments to $L_{1}$. As a consequence $\mathfrak{M} \forall_{\varepsilon}$ ist $(1, p \supset r)$. Soundness of PLC and (7) entail that $\mathfrak{M} \forall_{\varepsilon} \bigwedge_{b r \in b r} l a(b r)$, and therefore, that there is an $n \leq N$ such that

$$
\mathfrak{M} \models_{\varepsilon} \bigwedge_{k=1}^{K_{n}} i s t\left(i_{n k}, \phi_{n k}\right) \quad \text { and } \quad \mathfrak{M} \forall_{\varepsilon} i \operatorname{ist}\left(j_{n}, \psi_{n}\right)
$$

The left part of (8) states that each $\phi_{n k}$ (with $1 \leq k \leq K_{n}$ ) is a tautology, as it must be true in all the assignments in $\mathfrak{M}\left(i_{n k}\right)$. As a consequence we have that

$$
\vdash_{\varepsilon} \bigwedge_{k=1}^{K_{n}} i s t\left(i_{n k}, \phi_{n k}\right)
$$


The right part of (8) states that there is an assignment $\boldsymbol{v} \in \mathfrak{M}\left(j_{n}\right)$ such that $\boldsymbol{\forall} \not \psi_{n}$, i.e., $\psi_{n}$ is not a tautology. Let us consider two cases $n \leq M$, and $n>M$. In the first case we have, due to the definiton of $l a\left(b r_{12}\right)$, we have that

$$
\vdash_{\varepsilon} l a\left(b r_{12}\right) \supset\left(\bigwedge_{k=1}^{K_{n}} i s t\left(i_{n k}, \phi_{n k}\right) \supset \operatorname{ist}\left(j_{n}, \psi_{n}\right)\right)
$$

while, in the second one we have:

$$
\vdash_{\varepsilon} l a\left(b r_{21}\right) \supset\left(\bigwedge_{k=1}^{K_{n}} i s t\left(i_{n k}, \phi_{n k}\right) \supset \operatorname{ist}\left(j_{n}, \psi_{n}\right)\right)
$$

By applying Modus Ponens to (10) and (9), or to (11) and (9), we obtain one of the following two consequences:

$$
\vdash_{\varepsilon} l a\left(b r_{12}\right) \supset \operatorname{ist}\left(j_{n}, \psi_{n}\right) \quad \text { or } \quad \vdash_{\varepsilon} l a\left(b r_{21}\right) \supset \operatorname{ist}\left(j_{n}, \psi_{n}\right)
$$

If the equivalence holds we would have that, either $\vdash_{b r_{12}} j_{n}: \psi_{n}$ or $\vdash_{b r_{21}} j_{n}: \psi_{n}$, while $\psi_{n}$ is not a tautology. But this is a contradiction.

Lifting axioms are not the only possible ist-formulas. There are ist-formulas, as for instance $\neg i s t(i, \phi)$ or $i s t(i, \phi) \supset i s t(j, \psi) \vee i s t(k, \theta)$, which are not lifting axioms but could be used to represent the compatibility relation formulated by bridge rules. So the question arises of whether bridge rules can be encoded by generic $i s t$-formulas in some external context $\varepsilon$. In the following we show that this is the case for MCSs with a finite number of contexts and with finite languages.

Theorem 3. There is a transformation a(.) from finite sets $\boldsymbol{b r} \in \mathbb{B R}_{I}$ of bridge rules to ist-axioms, and a context $\varepsilon$ such that:

$$
\begin{gathered}
i_{1}: \phi_{1}, \ldots, i_{n}: \phi_{n} \vdash_{\boldsymbol{b r}} i: \phi \\
\text { if and only if } \\
\vdash_{\varepsilon} a(\boldsymbol{b r}) \supset \operatorname{ist}\left(i_{1}, \phi_{1}\right) \wedge \ldots \wedge i \operatorname{sit}\left(i_{n}, \phi_{n}\right) \supset \operatorname{ist}(i, \phi)
\end{gathered}
$$

Proof. The proof is constructive, i.e., we define the transformation $a($.$) for each set of$ bridge rules. The definition of $a(\boldsymbol{b r})$ passes through a syntactic encoding of the LMSmodels for $\boldsymbol{b r}$.

Let $C$ be a LMS-model (i.e. a set of chains), the set of PLC-models $\mathfrak{M}_{C}$ corresponding to $C$ is defined as follows:

$$
\mathfrak{M}_{C}=\left\{\begin{array}{l|l}
\mathfrak{M}_{C^{\prime}} & \begin{array}{l}
C^{\prime} \text { is a subset of } C \text { such that for any } i \in I, \mathfrak{M}(i)= \\
\bigcup_{c \in C^{\prime}} c_{i}
\end{array}
\end{array}\right\}
$$

Let $\mathbf{C}$ be the set of LMS-models for $\boldsymbol{b r}$. The set $\mathfrak{M}_{\mathbf{C}}$ is defined as $\bigcup_{C \in \mathbf{C}} \mathfrak{M}_{C}$. Let us prove that the logical consequence defined by $\mathbf{C}$ can be represented by valid formulas in the set of models $\mathfrak{M}_{\mathbf{C}}$, i.e., that:

$$
\begin{gathered}
i_{1}: \phi_{1}, \ldots, i_{n}: \phi_{n}=\mathbf{C} i: \phi \\
\text { if and only if for all } \mathfrak{M}_{\in} \in \mathfrak{M}_{\mathbf{C}} \\
\mathfrak{M} \models_{\varepsilon} i \operatorname{ist}\left(i_{1}, \phi_{1}\right) \wedge \ldots \wedge i s t\left(i_{n}, \phi_{n}\right) \supset i s t(i, \phi)
\end{gathered}
$$


Suppose that $i_{1}: \phi_{1}, \ldots, i_{n}: \phi_{n} \models_{\mathbf{C}} i: \phi$. Let $\mathfrak{M}_{C^{\prime}} \in \mathfrak{M}_{\mathbf{C}}$, with $C^{\prime} \subseteq C \in \mathbf{C}$. Suppose that $\mathfrak{M}_{C^{\prime}} \models_{\varepsilon} i s t\left(i_{k}, \phi_{k}\right)$ for any $1 \leq k \leq n$. This implies that for all $c \in C^{\prime}, c_{i_{k}}=\phi_{k}$. From the hypothesis we have that $c_{i}=\phi$, and therefore that $\mathfrak{M}_{C^{\prime}} \models_{\varepsilon}$ ist $(i, \phi$,).

Vice-versa, let us prove that $\mathfrak{M}=_{\varepsilon}$ ist $\left(i_{1}, \phi_{1}\right) \wedge \ldots \wedge i s t\left(i_{n}, \phi_{n}\right) \supset$ ist $(i, \phi)$ for all $\mathfrak{M} \in$ $\mathfrak{M}_{\mathbf{C}}$ implies that for any model $C$ of $\boldsymbol{b r}$ and for any chain $c \in C$, if $c_{i_{k}} \models \phi_{k}$ for $1 \leq k \leq n$, then $c_{i}=\phi$. Notice that, for any $c \in C \in \mathbf{C}$ we have that $\mathfrak{M}_{\{c\}} \in \mathfrak{M}_{\mathbf{C}}$. By definition (see equation (13)), $\mathfrak{M}_{\{c\}}$ is such that $\mathfrak{M}(i)=c_{i}$. By hypothesis we have that $\mathfrak{M}_{\{c\}}=$ $\operatorname{ist}\left(i_{1}, \phi_{1}\right) \wedge \ldots \wedge i s t\left(i_{n}, \phi_{n}\right) \supset$ ist $(i, \phi)$, which implies that if $c_{i_{k}} \models \phi_{k}$ for all $1 \leq k \leq n$, then $c_{i}=\phi$.

To define $a(\boldsymbol{b r})$ we proceed as follows: for any PLC model $\mathfrak{M} \in \mathfrak{M}_{\mathbf{C}}$ we find a formula $\phi_{\mathfrak{M}}$, that axiomatizes exactly $\mathfrak{M}$. Then the axiomatization of $\mathfrak{M}_{\mathbf{C}}$ can be obtained by the disjunction of all the axiomatization $\phi_{\mathfrak{M}}$ associated to each single PLC-model $\mathfrak{M}$ of $\mathfrak{M}_{\mathbf{C}}$ (this definition is possible because $\mathfrak{M}_{\mathbf{C}}$ is finite).

Let $\mathfrak{M} \in \mathfrak{M}_{\mathbf{C}}$, and let $\phi_{\mathfrak{M}}$ be the following formula

$$
\bigwedge_{i \in I}\left(i s t\left(i, \bigvee_{v \in \mathfrak{M}(i)} \phi_{v}\right) \wedge \bigwedge_{v \in \mathfrak{M}(i)} \neg i s t\left(i, \neg \phi_{v}\right)\right)
$$

where $\phi_{v}$ is the conjunction of all the literals verified by the assignment $v$. (15) is a finite formula, for the set $I$ of context is finite and the set of literals in each context is finite too. By adding (15) as axioms in the context $\varepsilon$ we obtain an PLC that is satisfied only by the model $\mathfrak{M}$. Let

$$
a(\boldsymbol{b} r)=\bigvee_{\mathfrak{M} \in \mathfrak{M}_{\mathbf{C}}} \phi_{\mathfrak{M}}
$$

Let us now prove the equivalence (12). By soundness and completeness of $\boldsymbol{b} \boldsymbol{r}, i_{1}$ : $\phi_{1}, \ldots, i_{n}: \phi_{n} \vdash_{\boldsymbol{b r}} i: \phi$ holds if and only if

$$
i_{1}: \phi_{1}, \ldots, i_{n}: \phi_{n} \models_{\mathbf{C}} i: \phi
$$

By (14), we have that (16) holds if and only if for all $\mathfrak{M} \in \mathfrak{M}_{\mathbf{C}}$,

$$
\mathfrak{M} \models_{\varepsilon} i s t\left(i_{1}, \phi_{1}\right) \wedge \ldots \wedge i s t\left(i_{n}, \phi_{n}\right) \supset i s t(i, \phi)
$$

By construction of $a(\boldsymbol{b r}), \mathfrak{M} \models_{\varepsilon} a(\boldsymbol{b r})$, if and only if $\mathfrak{M} \in \mathfrak{M}_{\mathbf{C}}$. This implies that (17) holds if and only if

$$
\models_{\varepsilon} a(\boldsymbol{b r}) \supset i s t\left(i_{1}, \phi_{1}\right) \wedge \ldots \wedge i s t\left(i_{n}, \phi_{n}\right) \supset i s t(i, \phi)
$$

Finally, soundness and completeness of PLC implies that (18) holds if and only if $\vdash_{\varepsilon}$ $a(\boldsymbol{b r}) \supset i \operatorname{ist}\left(i_{1}, \phi_{1}\right) \wedge \ldots \wedge i s t\left(i_{n}, \phi_{n}\right) \supset i s t(i, \phi)$, which concludes our proof.

Theorem 3 shows that the translation from bridge rules to generic ist-formulas is possible. However, it is still open the question if a set of bridge rules can be translated into set of ist-formulas which are lifting axioms. Here the answer is negative. 
Theorem 4. There does not exist a transformation la (.) from finite sets $\boldsymbol{b r} \in \mathbb{B R}_{I}$ of bridge rules to a conjunction of lifting axioms, and a context $\varepsilon$ such that:

$$
\begin{gathered}
i_{1}: \phi_{1}, \ldots, i_{n}: \phi_{n} \vdash_{\boldsymbol{b r}} i: \phi \\
\text { if and only if } \\
\vdash_{\varepsilon} l a(\boldsymbol{b r}) \supset \operatorname{ist}\left(i_{1}, \phi_{1}\right) \wedge \ldots \wedge i s t\left(i_{n}, \phi_{n}\right) \supset \operatorname{ist}(i, \phi)
\end{gathered}
$$

Proof. The proof is by counterexample. Consider the following LMS/MCS composed of two languages $L_{1}$ and $L_{2}$ containing the single proposition $p$ and $q$ respectively. Consider the following set of bridge rules:

$$
\frac{1: \neg p}{2: q} b r_{12} \quad \frac{1: p}{2: \neg q} b r_{12}^{r} \quad \frac{2: \neg q}{1: p} b r_{21} \quad \frac{2: q}{1: \neg p} b r_{21}^{r} \quad \frac{1: \perp}{2: \perp} \perp_{12} \quad \frac{2: \perp}{1: \perp} \perp_{21}
$$

where all the rules but those indexed with $r$ are non restricted. The chains that satisfies the un-restricted bridge rules are:

$$
c=\langle p, \bar{q}\rangle, \quad d=\langle\bar{p}, q\rangle, \quad e=\langle p, q\rangle
$$

where $p$ denotes the model in which $p$ is true and $\bar{p}$ the model in which $p$ is false. Similarly for $q$ and $\bar{q}$. The compatibility relations that satisfy the restricted bridge rules are:

$$
\{c\},\{d\},\{e\},\{c, e\}, \quad\{d, e\}
$$

Following the definitions given in the proof of Theorem 3 one can see that the istformulas associated to the set of LMS-models above is equivalent to the following:

$$
\neg i s t(1, \perp) \wedge \neg i s t(2, \perp) \wedge(\operatorname{ist}(1, p) \vee \operatorname{ist}(2, q))
$$

Notice that the above formula cannot be reduced in the form of a conjunction of lifting axioms.

\section{Discussion}

In the previous section we have given two somehow opposite results: namely Theorem 2 and Theorem 3. Intuitively the former states that bridge rules cannot be transformed into lifting axioms, so that this translation composes; the latter states that finite sets of bridge rules can be translated into a finite sets of ist-formulas. This two results constitutes two boundaries within which one can look for further correspondence results.

Theorem 2 states that a set of bridge rules cannot be translated into a set of lifting axiom simply by translating each single bridge rule into a lifting axiom. This is intuitively due to the fact that bridge rules allows for inter-leaving of local reasonings, while lifting axioms do not. By inter-leaving of local reasonings we mean the reasoning pattern composed by a sequence of chunks of local reasoning. This reasoning pattern

allow for cyclic contextual reasoning. For instance, one starts in a context $\bar{\kappa}_{1}$ switches in a context $\bar{\kappa}_{2}$ then, switch back in the context $\bar{\kappa}_{1}$ and then again in the context $\bar{\kappa}_{2}$. 
Consider the bridge rules given in the counter-example of the proof of Theorem 2, plus the bridge rule:

$$
\frac{1: p \supset r}{2: s} b r_{12}^{\prime}
$$

An example of inter-leaving of local reasonings is the following proof of $2: s$.

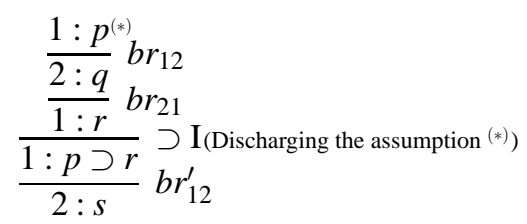

PLC does not support inter-leaving of local reasonings. The reasoning pattern implemented in PLC, instead, is "bottom up combination of local reasonings" in a tower of transcendent contexts. In this reasoning pattern one starts from the bottom of a tower of contexts, he locally reasons in a (set of) context(s), say in the context denoted by the sequence $\kappa_{1} \ldots \kappa_{n} \kappa$, then he transcends to by (CS) to the context $\kappa_{1} \ldots \kappa_{n}$ and he locally reasons there (e.g., by using the lifting axioms), then he transcends again to $\kappa_{1} \ldots \kappa_{n-1}$. Eventually, he stops at some point of the tower. Theorem 2 shows that "inter-leaving of local reasonings" cannot be reduced to "bottom-up combination of local reasonings + lifting axioms".

Theorem 3, instead, provides a way to translate LMS/MCS into PLC. Furthermore, the counterexample provides in Theorem 4 show that the one proposed in Theorem 3 is the "simplest" translation, i.e., that any other translation cannot be reduced to a conjunction of lifting axioms. If one wants to rewrite bridge rules into lifting axioms he has to take into account the following two points:

1. in embedding LMS/MCS into PLC, bridge rules are not directly translated into implications, as one could expect. For instance the MCS containing the bridge rules (4) are not translated into the axioms of the form ist $(1, p) \supset$ ist $(2, q)$ and ist $(2, q) \supset$ ist $(1, p)$ as shown by Theorem 2. Indeed, the PLC formalizing the bridge rules (4) is not computed by a direct (syntactic) translation of the bridge rules of MCS. The axioms (15) are determined by enumerating all the LMS-models of (4) and by axiomatizing them in a PLC-formula. This is not a problem of our translation, indeed any alternative translation which is equivalent to the axiom (15) with more than two contexts cannot be reduced to a set of lifting axioms.

2. the above translation is not compositional. This means that, if $\mathrm{PLC}_{1}$ and $\mathrm{PLC}_{2}$ are the representations of $\mathrm{MCS}_{1}$ and $\mathrm{MCS}_{2}$ respectively, then the translation of $\mathrm{MCS}_{1} \cup \mathrm{MCS}_{2}$ (i.e., the MCS containing the axioms and the bridge rules of both $\mathrm{MCS}_{1}$ and $\mathrm{MCS}_{2}$ ) cannot be defined as the union of the axioms of PLC 1 and $\mathrm{PLC}_{2}$.

\section{Conclusions}

This paper concludes the technical and conceptual comparison between LMS/MCS and PLC we started in [3]. The results presented in this paper will help clarify the technical and conceptual differences between the two approaches, by showing how bridge rules can be represented in lifting axioms or in ist-formulas. In particular we have shown that: 
1. Bridge rules cannot be translated into lifting axioms;

2. sets of bridge rules can be translated into set of ist-formulas which cannot be reduced to a conjunction of lifting axioms.

We stress the fact that the two formalisms do not provide equivalent solutions, even if they share some of the intuitive motivations for having a formal theory of context in AI. The technical results we provide in the previous paper [3] and in this paper allow us to justify the conclusion that LMS/MCS is more general than PLC, and that it captures some patterns of contextual reasoning in a more intuitive and straightforward way. Moreover, in our opinion, the restrictions needed to reconstruct LMS/MCS in PLC have a significant impact on the appropriateness of PLC to capture the intuitive desiderata of a logic of context in AI.

\section{Acknowledgements}

We thank Massimo Benerecetti, Chiara Ghidini and Fausto Giunchiglia for the useful discussions on the paper. We also want to thank the anonymous reviewers for the useful comments, and in particular the reviewer who suggested to avoid some clumsiness in the paper by discussing some of the problems in terms of relative interpretations between logics [13]. For lack of time, we could not take the advice in this paper, but we plan to explore this idea in future versions of the paper.

\section{References}

1. V. Akman, P. Bouquet, R. Thomason, and R.A. Young, editors. Modeling and Using Context, volume 2116 of Lecture Notes in Artifi cial Intelligence. Springer Verlag, 2001. Proceedings of CONTEXT'2001 - Third International and Interdisciplinary Conference on Modeling and Using Context (27-30 July 2001, Dundee, Scotland).

2. P. Bouquet and F. Giunchiglia. Reasoning about theory adequacy: A new solution to the qualification problem. Fundamenta Informaticae, 23(2-4):247-262, June,July,August 1995. Also IRST-Technical Report 9406-13, IRST, Trento, Italy.

3. P. Bouquet and L. Serafini. Two formalizations of context: a comparison. In Akman et al. [1], pages 87-101. Proceedings of CONTEXT'2001 - Third International and Interdisciplinary Conference on Modeling and Using Context (27-30 July 2001, Dundee, Scotland).

4. S. Buvač, V. Buvač, and I.A. Mason. Metamathematics of Contexts. Fundamentae Informaticae, 23(3), 1995.

5. S. Buvač and Ian A. Mason. Propositional logic of context. In R. Fikes and W. Lehnert, editors, Proc. of the 11th National Conference on Artificial Intelligence, pages 412-419, Menlo Park, California, 1993. American Association for Artificial Intelligence, AAAI Press.

6. G. Criscuolo, F. Giunchiglia, and L. Serafini. A Foundation for Metareasoning, Part I: The proof theory. Technical Report 0003-38, IRST, Trento, Italy, 2000. To be published in the Journal of Logic and Computation.

7. C. Ghidini and F. Giunchiglia. Local Models Semantics, or Contextual Reasoning = Locality + Compatibility. Artifi cial Intelligence, 127(2):221-259, April 2001.

8. F. Giunchiglia. Contextual reasoning. Epistemologia, special issue on I Linguaggi e le Macchine, XVI:345-364, 1993. Short version in Proceedings IJCAI'93 Workshop on Using Knowledge in its Context, Chambery, France, 1993, pp. 39-49. Also IRST-Technical Report 9211-20, IRST, Trento, Italy. 
9. F. Giunchiglia and L. Serafini. Multilanguage hierarchical logics or: how we can do without modal logics. Artifi cial Intelligence, 65(1):29-70, 1994. Also IRST-Technical Report 911007, IRST, Trento, Italy.

10. R.V. Guha. Contexts: a Formalization and some Applications. Technical Report ACT-CYC423-91, MCC, Austin, Texas, 1991.

11. J. McCarthy. Notes on Formalizing Context. In Proc. of the 13th International Joint Conference on Artifi cial Intelligence, pages 555-560, Chambery, France, 1993.

12. D. Prawitz. Natural Deduction - A proof theoretical study. Almquist and Wiksell, Stockholm, 1965.

13. A. Tarski, A. Mostowski, and R.M. Robinson. Undecidable theories. North-Holland, 1968. 